Communications in Physics, Vol.22, No. 2 (2012), pp. 103-110

\title{
PHASE STRUCTURE OF LINEAR SIGMA MODEL WITH NEUTRALITY CONSTRAINT (II)
}

\author{
TRAN HUU PHAT \\ Vietnam Atomic Energy Commission \\ and \\ Dong Do University \\ NGUYEN VAN THU \\ Institute of Nuclear Science and Technique \\ and \\ Department of Physics, Hanoi University of Education II
}

\begin{abstract}
The phase structure of the linear sigma model with constituent quarks and the electric neutrality is systematically studied in the mean field theory. It results that in the chiral limit, as function of $T$ and chemical potential $\mu$, the pion condensate undergoes a first-order phase transition. In the physical world, the phase diagram of chiral condensate exhibits a first-order phase transition, which ends at a critical end point (CEP) for $\alpha<0.3$, where $\alpha$ is the fraction of $\pi^{+}$contribution to the condensate.
\end{abstract}

\section{INTRODUCTION}

It is known that the study of the phase structure of quantum chromodynamics (QCD) turns out to be a very hot subject attracted more and more attention, both experimentally and theoretically [1]. Many aspects of in-medium effects such as the chiral restoration at high baryon density, the in-medium properties of hadrons, the pion condensation in dense matter and so on are of special interest. The high energy heavy-ion collisions are presently the powerful machinery to generate hot and dense hadronic matter, and, therefore, they create a good chance for exploring the phase structure of QCD at extreme conditions. It is commonly accepted that the chiral restoration phase transition accompanies the confinement - deconfinement phase transition at the same critical temperature. The recent experimental data of RHIC, collected for high-temperature and low-baryon density region, provide clear signals on the confinement-deconfinement phase transition. This region is also one of the research problems to be carried out at LHC. The experimental research in high-baryon density region is planned to be implemented in the future heavy-ion collision experiments at RHIC, FAIR and NICA.

The first principle calculations of lattice QCD at finite baryon density is rather problematic due to the sign puzzle. In this respect, one has to invoke different effective models to address this issue.

We know that heavy nuclei can be considered as nuclear matter with finite isospin density due to Coulomb interactions and the isospin-dependent quantities of isotopically 
asymmetric nuclear matter, such as the in-medium nucleon-nucleon potential, the equations of state and, in particular, the nuclear symmetry energy plays very important role in understanding a lot of interesting astrophysical problems [2]- [4], dynamics of heavy-ion reactions at intermediate energies [5]- [8], the structure of neutron-rich nuclei, and the nuclei close to the drip-line [9]- [14]. Hence, dealing with QCD at finite isospin chemical potential is of special significance, too. The lattice-QCD (LQCD) simulations $[15,16]$ at finite isospin chemical potential, finite temperature and low baryonic density were smoothly implemented and conjectured that the charged pions condense at high isospin density and low temperature, and also suggested that the transition to a Bose-Einstein condensed state coincides with the deconfinement phase transition to quark-gluon plasma at low isospin chemical potential. The pion condensation and the related phase diagrams of two-flavor QCD have been investigated by means of various QCD effective models: chiral perturbation theory [17]- [19], Nambu-Jona-Lasinio (NJL)-type models [20]- [23], linear sigma model [24]. We are aware that, among theories developed so far, the LQCD is the unique one based on first principle and the Polyakov-loop extended (PNJL) model is an extended version of the NJL model which respects approximately both mechanisms: chiral symmetry breaking and confinement. In this regard, perhaps, they provide a guideline for all studies of the phase structure.

Nowadays, the holographic QCD [25] emerges as a promised theory that is hopeful to explain satisfactorily all phenomena related to the non- perturbative region of QCD.

In the present paper the effect of the neutrality condition on the phase structure of the linear sigma model with constituent quarks is studied. We will closely follow Ref. [26] to consider the system given by the Lagrangian

$$
\begin{aligned}
& L=\bar{q}\left[i \gamma^{\mu} \partial_{\mu}-m_{q}-g\left(\sigma+i \gamma^{5} \vec{\tau} \vec{\pi}\right)\right] q+\mu \bar{q} \gamma^{0} q+L_{L S M}, \\
& L_{L S M}=\frac{1}{2}\left[\partial_{\alpha} \sigma \partial^{\alpha} \sigma+\partial_{\alpha} \vec{\pi} \partial^{\alpha} \vec{\pi}\right]+2 i \mu_{I}\left(\pi_{1} \partial_{0} \pi_{2}-\pi_{2} \partial_{0} \pi_{1}\right)-U, \\
& U=\frac{m^{2}}{2}\left(\sigma^{2}+\vec{\pi}^{2}\right)-\frac{\mu_{I}^{2}}{2}\left(\pi_{1}^{2}+\pi_{2}^{2}\right)+\frac{\lambda^{2}}{4}\left(\sigma^{2}+\vec{\pi}^{2}\right)^{2}-\epsilon f_{\pi} m_{\pi}^{2} \sigma,
\end{aligned}
$$

where $q, \sigma$ and $\vec{\pi}$ are respectively the field operators of quarks, sigma meson and pion; $m_{\pi}$ and $f_{\pi}$ are respectively pion mass and pion decay constant in vacuum; $\mu$ and $\mu_{I}$ denote the baryon and isospin chemical potentials, $m_{q}$ is current quark mass and $\epsilon=0,1$.

The paper is structured as follows. In Section II the basic formulae and phase diagram of the system (1) are considered, respectively, in the chiral limit and the physical world. The conclusion and discussion are given in Section III.

\section{THE BASIC FORMULAE AND PHASE DIAGRAM}

In our previous works [27] we found the gap equations

$$
\begin{aligned}
& {\left[m^{2}+\lambda^{2}\left(u^{2}+v^{2}\right)\right] u-\epsilon f_{\pi} m_{\pi}^{2}+\frac{\partial \Omega_{q \bar{q}}}{\partial u}=0,} \\
& {\left[-\mu_{I}^{2}+m^{2}+\lambda^{2}\left(u^{2}+v^{2}\right)\right] v+\frac{\partial \Omega_{q \bar{q}}}{\partial v}=0,}
\end{aligned}
$$


and proved that the quark densities fulfill the equality

$$
\rho_{u}+\rho_{d}=\rho,
$$

in which $\partial \Omega_{q \bar{q}} / \partial u, \partial \Omega_{q \bar{q}} / \partial v$ and $\rho$ are given in Eqs. (9), (10) and (12) of [27].

Next we consider the electric neutrality. The bulk matter of stars is electrically neutral and the color neutrality is automatically satisfied in our case. So, the electric neutrality has to be incorporated into consideration.

In addition, matter must be stable under the weak processes like

$$
\mathrm{d} \rightarrow \mathrm{u}+\mathrm{e}^{-}+\widetilde{\nu}_{e} .
$$

The chemical potential of neutrinos is zero if we assume that the neutrinos can leave the system, then the chemical equilibrium requires

$$
\mu_{u}-\mu_{d}=-\mu_{e} .
$$

in which $\mu_{e}$ is the electron chemical potential. The chemical equilibrium related to the presence of charged pion condensate imposes the condition

$$
\begin{aligned}
& \mu_{u}-\mu_{d}=\mu_{I}, \\
& \mu_{e}=-\mu_{I} .
\end{aligned}
$$

Let us remember that, in addition to Eq.(4a), we already have another relation

$$
\mu_{u}+\mu_{d}=\mu,
$$

which together with (4a) lead to

$$
\rho_{u}+\rho_{d}=\rho, \rho_{u}-\rho_{d}=\rho_{I} .
$$

The electric neutrality means that the total charge density in condensed phase has to be zero, namely,

$$
Q=\sum_{B=u, d} q_{B} \rho_{B}+\alpha \rho_{I}-(1-\alpha) \rho_{I}+\rho_{e^{+}}=0
$$

here $q_{B}$ is the charge of quark $\mathrm{B}, 0 \leq \alpha \leq 1$ means the portion of $\pi^{+}$condensate in the charged pion condensate, $(1-\alpha)$ is the portion of $\pi^{-}$condensate in the charged pion condensate and $\rho_{e^{+}}$denotes the positron density and

$$
\rho_{I}=\mu_{I} v^{2} \text {. }
$$

Taking into account (4b) Eq. (6) is rewritten

$$
\sum_{B=u, d} q_{B} \rho_{B}=(1-2 \alpha) \rho_{I}+\left.\left(\frac{\partial \Omega_{e}}{\partial \mu_{e}}\right)\right|_{\mu_{e}=-\mu_{I}},
$$

where $\Omega_{e}$ is the effective potential of electron

$$
\begin{aligned}
\Omega_{e} & =-2 \int \frac{d^{3} \vec{p}}{(2 \pi)^{3}}\left\{E_{p}+T \ln \left[1+e^{-\beta\left(E_{p}-\mu_{e}\right)}\right]+T \ln \left[1+e^{-\beta\left(E_{p}+\mu_{e}\right)}\right]\right\}, \\
E_{p} & =\sqrt{\vec{p}^{2}+m_{e}^{2}}
\end{aligned}
$$


derived from the Lagrangian

$$
L_{e}=\bar{\psi}_{e}\left(i \gamma^{\mu} \partial_{\mu}+e \gamma^{0} \mu_{e}-m_{e}\right) \psi_{e},
$$

and $\psi_{e}, e$ and $m_{e}$ denote respectively the electron field, the electron charge and the electron mass.

Inserting (5) into (7) we arrive at the neutrality constraint which determines $\mu$ as a function of $\mu_{I}$ and vice versa. This implies that, from now on, our system depends upon only two parameters, for example $T$ and $\mu$. In the remainder, we neglect the electron mass for simplicity.

Now we consider how the above calculated phase diagrams change under the neutrality constraint. In order to proceed to the numerical computation the values of the model parameters are usually chosen as $m_{q}=5.5 \mathrm{MeV}, f_{\pi}=93 \mathrm{MeV}, m_{\pi}=138 \mathrm{MeV}$, sigma mass $m_{\sigma}=500 \mathrm{MeV}$ and the coupling constant $g$ is determined to be $g=3.3$. We are ready to implement the numerical study in two different cases with the aid of Mathematica [28].

\section{II.1. The chiral limit $\epsilon=0$}

In this case the chiral condensate vanishes and solving simultaneously the gap equation (3) and the neutrality equation (7) provides in Fig.1(a) the evolution of the pion condensate versus $T$ at $\mu=100 \mathrm{MeV}$ and $\alpha=0$ (solid line), 1/4 (dashed line), 1/2 (dotted line). All lines have the same meeting point at $T=96.7 \mathrm{MeV}$. The pion condensation is a first-order phase transition. In Fig.1(b) we show the evolution of the pion condensate versus $\mu$ at $T=0$ and $\alpha=0$ (solid line), $1 / 4$ (dashed line), $1 / 2$ (dotted line). All lines have the same meeting point at $\mu=205.3 \mathrm{MeV}$. Evidently, the phase transition is the first-order everywhere. The corresponding phase diagram in the $(T, \mu)$-plane is plotted in Fig.2. The NJL model calculations [12] claim that a first-order line ends at a critical point and the transition is second-order all the way to $\mu=0$.

\section{II.2. The physical world $\epsilon=1$}

1- We first consider the case $m_{\pi}<\mu_{I}$ where both chiral and pion condensates are non-vanishing. Simultaneously calculating the gap equations (2), (3) and the neutrality equation (7) it follows that

- In Fig.3(a) is shown the graphs representing the evolutions of pion condensate against $T$ at $\mu_{I}=200 \mathrm{MeV}$ and $\alpha=0$ (solid line), 0.25 (dashed line), 0.3 (dotted line), all lines have the same meeting point at $T=52.5 \mathrm{MeV}$. Fig.3(b) displays the $\mu$ dependence of the pion condensate at $T=50 \mathrm{MeV}$ and $\alpha=0$ (solid line), 0.25 (dashed line), 0.3 (dotted line), all lines have the same meeting point at $\mu=234 \mathrm{MeV}$. Evidently, in both cases a first-order phase transition occurs everywhere. The analyses based on NJL model [22] assert that no charged pion condenses in the physical world.

- Making use of the effective potential $\Omega(M)$ we show in Fig.4 the phase diagram of chiral condensate taken at several values of $\alpha$. From the bottom to top the lines correspond to $\alpha=0,0.25,0.3$. The solid line (dashed line) denotes the first-order (smooth) phase transition. $\mathrm{M}(T=347 \mathrm{MeV}, \mu=662 \mathrm{MeV})$ and $\mathrm{N}(T=373.7 \mathrm{MeV}, \mu=1232 \mathrm{MeV})$ are the second-order critical endpoints. For $\alpha=0.3$ the transition is smooth everywhere. The 


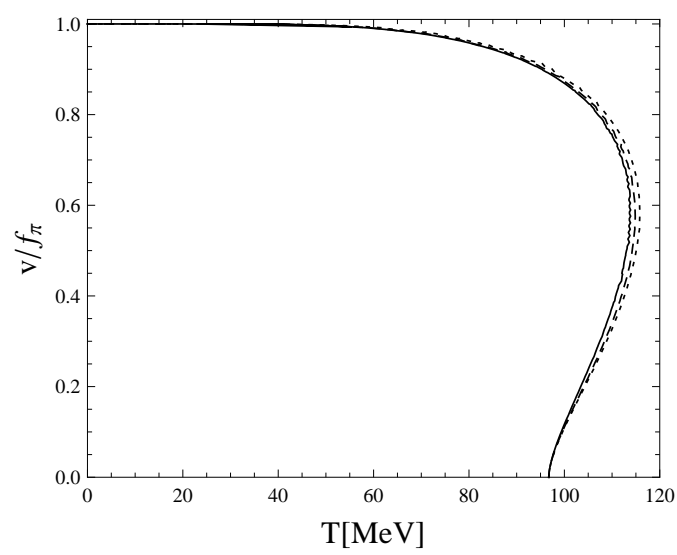

(a)

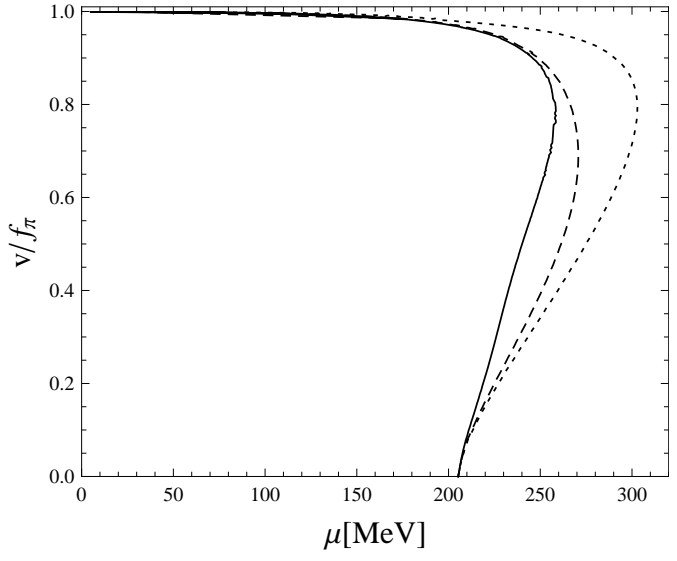

(b)

Fig. 1. The pion condensate as a function of $T$ (Fig.1(a)) at $\mu=100 \mathrm{MeV}$ and of $\mu$ at $T=50 \mathrm{MeV}$ (Fig.1(b)) in the chiral limit for neutral matter. The solid, dashed and dotted lines correspond to $\alpha=0,1 / 4,1 / 2$.

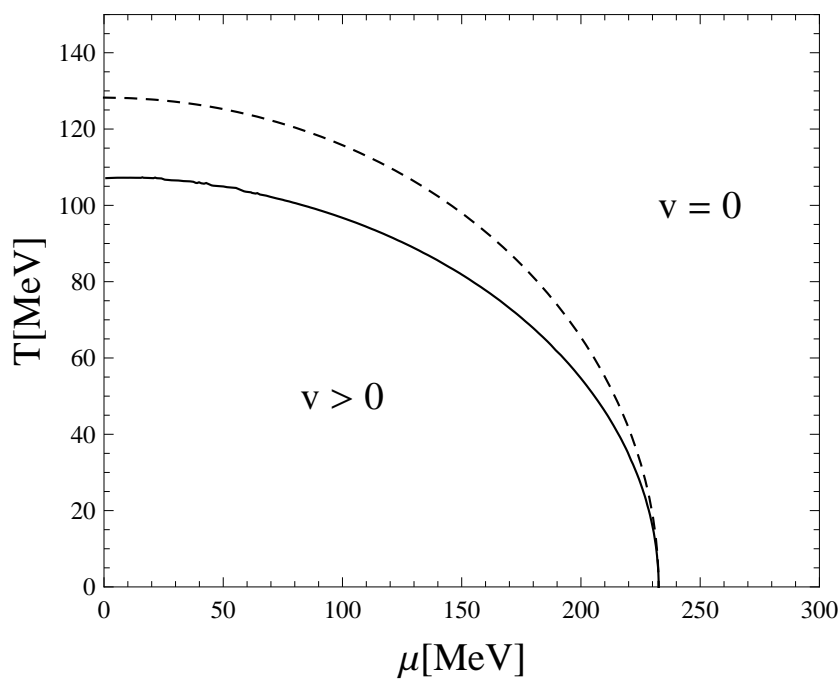

Fig. 2. The phase diagram of pion condensate in the $(T, \mu)$-plane for neutral matter in chiral limit. In order to have some comparison, we add to the figure the dashed line representing the phase diagram of pion condensate in the $(T, \mu)$-plane at $\mu_{I}=232.576 \mathrm{MeV}$ when the neutrality condition is not used.

present scenario is quite different from what was derived in Ref. [22] which claims that the chiral condensate decreases with increasing $T$ and $\mu$, but never vanishes.

2- In the case $m_{\pi}>\mu_{I}$ the pion condensate vanishes and we receive in Figs.5(a) and $5(\mathrm{~b})$ the evolution of the chiral condensate against $T$ for $\mu_{I}=0$ (solid line), 100 


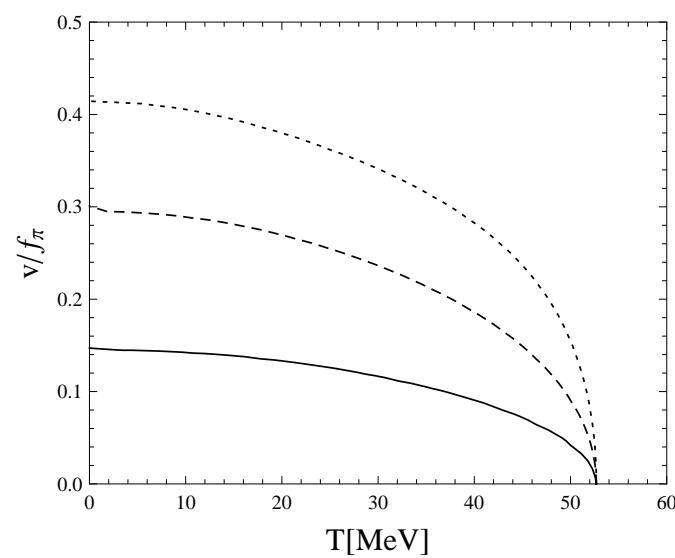

(a)

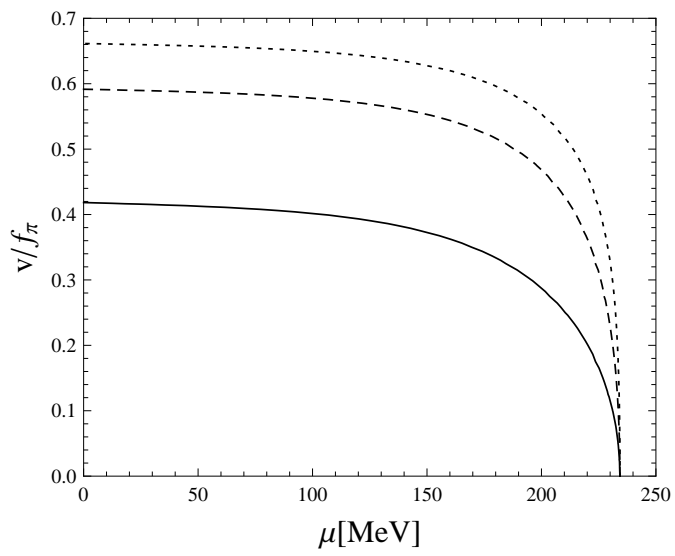

(b)

Fig. 3. The pion condensate as a function of $T$ (Fig.3(a)) at $\mu_{I}=200 \mathrm{MeV}$ and of $\mu$ (Fig.3(b)) at $T=50 \mathrm{MeV}$ in the physical world for neutral matter. The solid, dashed and dotted lines correspond to $\alpha=0,0.25,0.3$.

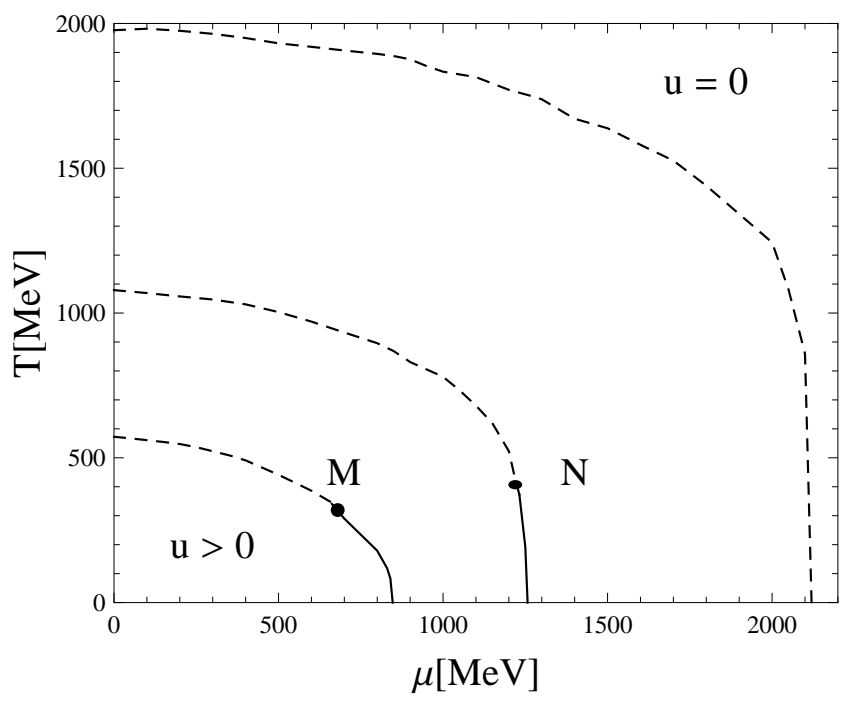

Fig. 4. The phase diagram of chiral condensate in $(T, \mu)$-plane for neutral matter in the physical world. From the bottom to top the graphs correspond to $\alpha=0,0.25,0.3$. The solid line (dashed line) denotes first-order (smooth) phase transition. $\mathrm{M}(662,346.8) \mathrm{MeV}$ and $\mathrm{N}(1232,373,6) \mathrm{MeV}$ are second order critical end points. For $\alpha=0.3$ only smooth phase transition occurs.

$\mathrm{MeV}$ (dashed line) and the phase diagram in the $\left(T, \mu_{I}\right)$-plane. They exhibit the smooth transition everywhere. 


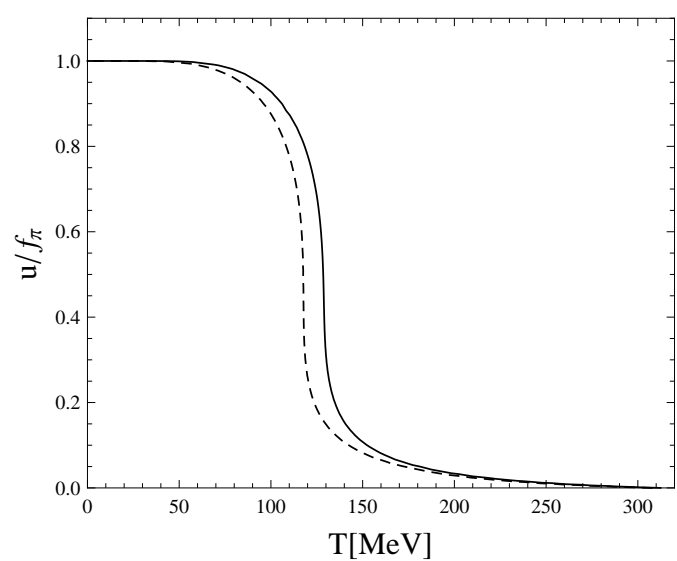

(a)

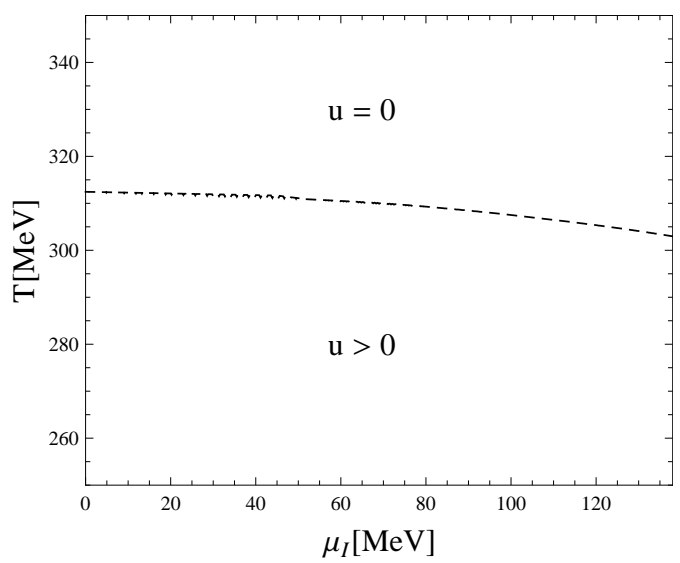

(b)

Fig. 5. In the region $\mu_{I}<m_{\pi}$ : Fig.5(a) shows $T$ dependence of $u$ for neutral matter (solid and dashed lines correspond to $\mu_{I}=0,100 \mathrm{MeV}$ ) and Fig.5(b) shows the phase diagram of chiral condensate in $\left(T, \mu_{I}\right)$ plane for neutral matter.

\section{CONCLUSION AND DISCUSSION}

In this paper the phase structure of the linear sigma model with constituent quarks was investigated systematically within the neutrality condition and the main results are in order.

a- The neutrality constraint was established.

b- The phase diagrams of the chiral condensate depends strongly on the ratio between the $\pi^{+}$and $\pi^{-}$contributions to the condensate. Meanwhile, the phase diagram of pion condensate does not depend on $\alpha$.

c- The phase diagram of the chiral condensate in the $(T, \mu)$-plane exhibits a first order phase transition which ends at a CEP for $\alpha<0.3$.

It is necessarily pointed out that the electric neutrality condition makes basic changes in the phase diagram of pion and chiral condensate.

\section{ACKNOWLEDGMENT}

N.V.Thu expresses his sincere thanks to the Vietnam Atomic Energy Commission and the Institute of Nuclear Science and Technique for the hospitality extended to him during his $\mathrm{PhD}$ study.

This paper is financially supported by the Vietnam National Foundation for Science and Technology Development (NAFOSTED).

\section{REFERENCES}

[1] M. A. Stephanov, Prog. Theor. Phys. Suppl. 153 (2004) 139; J. Wambach, Proceedings of International Workshop on Hot and Cold Baryonic Matter. Budapest, Aug. 15-20, 2010, Hungaria; D. H. Rischke, Proc. Part. Nucl. Phys. 52 (2004) 197.

[2] A. W. Steiner, M. Prakash, J. M. Lattimer, and P. J. Ellis, Phys. Rept. 411 (2005) 325. 
[3] C. H. Hee, Phys. Rept. 275 (1996) 255.

[4] I. Bombaci, in Isospin Physics in Heavy-Ion Collisions, edited by B. A. Li and Udo Schroeder, Nova Science Publishers, Inc., New York, 2001, pp.35-81.

[5] V. Baran, M. Colonna, V. Greco, and M. Di Toro, Phys. Rept. 410 (2005) 335.

[6] B. A. Li, L. W. Chen, and C. M. Ko, Phys. Rept. 464 (2008) 113.

[7] P. Danielewicz and Jenny Lee, Int. J. Mod. Phys. E18 (2009) 892.

[8] B. A. Li, C. M. Ko, and W. Bauer, Int. J. Mod. Phys. E7 (1998) 147.

[9] C. J. Horowitz and J. Piekarewicz, Phys. Rev. Lett. 86 (2001) 5647.

[10] S. Typel and B. A. Brown, Phys. Rev. C64 (2001) 027302.

[11] F. Hofmann, C. M. Keil, and H. Lenske, Phys. Rev. C 64, 034314 (2001).

[12] J. R. Stone, J. C. Miller, R. Koncewicz, P. D. Stevenson, and M. R. Strayer, Phys. Rev. C 68, 034324 (2003).

[13] A. W. Steiner and B. A. Li, Phys. Rev. C72 041601 (R) (2005).

[14] B. A. Brown, Phys. Rev. Lett. 85, 5296 (2000).

[15] J. B. Kogut and D. K. Sinclair, Phys. Rev. D64 (2001) 034508; ibid. D66 (2002) 034505. ibid. D70 (2004) 094501.

[16] S. Gupta, hep-lat/02005; P. de Forcrand, M. A. Stephanov, and U. Wenger, PoSLAT 2007, 237 (2007).

[17] D. T. Son and M. A. Stephanov, Phys. Rev. Lett. 86 (2001) 592.

[18] K. Splittorff, D. T. Son, and M. A. Stephanov, Phys. Rev. D64 (2001) 016003.

[19] K. Splittorff, D. Toublan, and J. J. Verbaarshot, Nucl. Phys. B639 (2002) 524.

[20] A. Barducci, R. Casalbuoni, G. Pettini, and L. Ravagli, Phys. Rev. D69 (2004) 096004; D. Ebert and K. G. Klimenko, J. Phys. G Part. Nucl. Phys. 32 (2006) 599; D. Ebert and K. G. Klimenko, Eur. Phys. J. C46 (2006) 771; S. Lawley, W. Bentz, and A. W. Thomas, Phys. Lett. B632 (2006) 495; L. He, Phys. Rev. D82 (2010) 096003.

[21] L. He, M. Jin, and P. Zhuang, Phys. Rev. D71 (2005) 116001.

[22] J. O. Andersen and L. Kyllingstad, J. Phys. G. Part. Nucl. Phys. 37 (2010) 015003.

[23] T. Sasaki, Y. Sakai, H. Kouno, and M. Yahiro, Phys. Rev. D82 (2010) 116004.

[24] J. O. Andersen, Phys. Rev. D75 (2007) 065011 ; J. O. Andersen and T. Brauner, Phys. Rev. D78 (2008) 014030.

[25] T. Sakai and S. Sugimoto, Prog. Theor. Phys. 113 (2005) 843; ibid. 114 (2006) 1083.

[26] D. K. Campell, R. F. Dashen, and J. T. Manassah, Phys. Rev. D12 (1975) 979; ibid. D12 (1975) 1010.

[27] Tran Huu Phat and Nguyen Van Thu, Comm. Phys. 22 (2012) 15-31.

[28] S. Wolfram, The Mathematica Book, 5th edition, Wolfram Media/Cambridge University Press, Cambridge , UK, 2003.

[29] H. Abuki, R. Anglani, R. Gatto, M. Pellicoro, and M. Ruggieri, arxiv: 0809.2658.

[30] J. W. Harris and B. Muller, Ann. Rev. Nucl. Part. Sci. 46 (1996) 71.

[31] V. Bernard, Ulf- G. Meissner, and I. Zahed, Phys. Rev. D36 (1987) 819.

[32] M. Buballa, Phys. Rept. 407 (2005) 205.

[33] M. Askawa and K. Yazaki, Nucl. Phys. A504 (1989) 668.

[34] T. Hatsuda and T. Kunihiro, Phys. Rept. 247 (1994) 221.

[35] M. A. Stephanov, Int. J. Mod. Phys. A20 (2005) 4387; hep-ph/0402115.

[36] R. V. Gavai and S. Gupta, Phys. Rev. D68 (2003) 034506 .

Received 14 October 2011. 